\title{
THE ANISOTROPY OF THE ELECTRICAL RESISTIVITY AND THERMAL EXPANSION OF SINGLE CRYSTAL DyNi
}

\author{
E. KAC̆MARČIKovÁa ${ }^{a}$, M. REIFFers ${ }^{a}$, M. MIHÁLIK ${ }^{a}$, J. Ková $^{a}$, \\ A. Menovsky ${ }^{b}$, J. Garcia Soldevilla ${ }^{c}$, A. Señas Pariente ${ }^{c}$ \\ AND J.C. GOMÉz SAL ${ }^{c}$ \\ ${ }^{a}$ Institute of Experimental Physics, Watsonova 47, 04353 Košice, Slovakia \\ ${ }^{b}$ Van der Waals-Zeeman Institute, University of Amsterdam \\ 1018 XE Amsterdam, Netherland \\ ${ }^{\circ}$ CITYMAC, Facultad de Ciencias, Universidad de Cantabria, 39005 Santander, Spain
}

The results of the measurements of temperature dependence of the electrical resistivity $\rho(T)$, thermal expansion $L(T)$ and susceptibility $\chi(T)$ on single crystal $\mathrm{DyNi}_{5}$ in the temperature range $4.2-300 \mathrm{~K}$ are reported. The maximum in $\rho(T)$ at $T_{\mathrm{N}}=12.2 \mathrm{~K}$ was observed which corresponds to the transition to antiferromagnetically ordered state. Taking into account the Bloch-Grüneisen formula for electron-phonon contribution $\rho_{\mathrm{ph}}(T)$ to $\rho(T)$ the anisotropic magnetic contribution $\rho_{\text {mag }}(T)$ was determined. We also observed the anisotropic behaviour of $L(T)$ at low temperatures.

PACS numbers: $72.15 . \mathrm{Eb}, 65.70 .+\mathrm{y}, 71.70 . \mathrm{Gm}$

The intermetallic $\mathrm{RNi}_{5}(\mathrm{R}=$ rare earths) compounds crystallize in the simple hexagonal structure and have been the subject of detailed studies during the last years [1]. Their magnetic properties are quite well understood and they are predominantly determined by the crystalline electric field (CEF) acting on $\mathrm{R}$ and the exchange interactions. In this series the $\mathrm{Ni}$ atoms are non-magnetic, although they are very close to the onset of $\mathrm{Ni}$ magnetism. However, till now there is only a few pieces of information about the anisotropy of the electrical transport in $\mathrm{LaNi}_{5}$ and $\mathrm{PrNi}_{5}$ [2] and only the information about the thermal expansion of $\mathrm{PrNi}_{5}$ [3]. From the heat capacity measurements follows that the intermetallic compound $\mathrm{DyNi}_{5}$ undergoes an antiferromagnetic ordering transition at $T_{\mathrm{N}}=11.6 \mathrm{~K}$ [4]. Most of information about CEF level scheme of $\mathrm{DyNi}_{5}$ has been obtained indirectly from specific heat [4], magnetization and susceptibility $[5,6]$ in the paramagnetic state. The electron-quasiparticle interaction function was measured by point-contact $(\mathrm{PC})$ spectroscopy above and below $T_{\mathrm{N}}[7]$. In order to study the anisotropy of the CEF contribution to the transport and thermal properties, for the first time we performed the measurements of the electrical resistivity and the 
thermal expansion coefficient $\alpha$ on the oriented single crystal of $\mathrm{DyNi}_{5}$. The susceptibility measurements were performed too.

The single crystal of a good quality was grown by the vertical floating zone method in an image furnace. The standard four probe d.c. method was used for the electrical resistivity measurements in the temperature range $4.2-300 \mathrm{~K}$ along 3 main crystallographical axis. The d.c. susceptibility measurements were performed in the vibrating sample magnetometer. The thermal expansion measurements were performed by resistive strain gauge technique within temperature range 4.2-300 $\mathrm{K}$ perpendicular and parallel to basal plane.

The temperature dependences of the electrical resistivity $\rho(T)$ of $\mathrm{DyNi}_{5}$ measured along the main crystallographical axis are shown in Fig. 1a. One could see the typical metallic behaviour. Taking into account the residual resistivities $\rho_{0}\left(\rho_{0}^{\boldsymbol{a}}=9.9 \mu \Omega \mathrm{cm}, \rho_{0}^{b}=11.7 \mu \Omega \mathrm{cm}\right.$ and $\left.\rho_{0}^{c}=8.5 \mu \Omega \mathrm{cm}\right)$, the following residual resistivity ratios $R R R=\rho(300 \mathrm{~K}) / \rho_{0}$ have been obtained: $R_{R R}=4.4$, $\mathrm{RRR}_{b}=4.0, \mathrm{RRR}_{c}=3.9$. The low temperature behaviour of the electrical resistivity $\left(\rho(T)-\rho_{0}\right)$ above and below $T_{\mathrm{N}}$ is presented in Fig. 1b. The clear maximum at $T_{\mathrm{N}}=12.2 \mathrm{~K}$ is connected with the transition to the antiferromagnetic state. This temperature is in a good agreement with previous data [4-6].
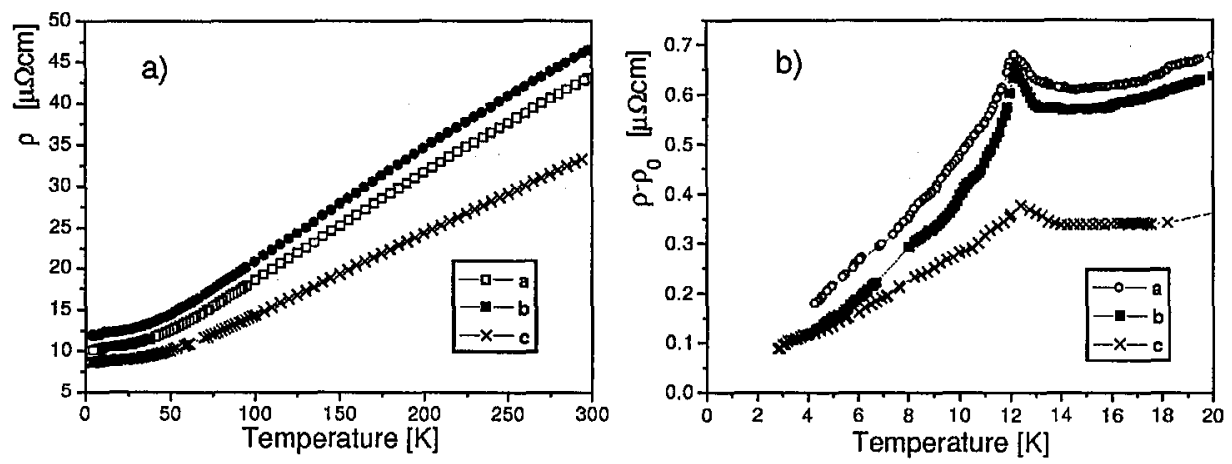

Fig. 1. The temperature dependences of the electrical resistivity of single crystal DyNi along main crystallographical axes (a). The low temperature detail is presented in (b).

In order to determine the magnetic contribution to the electrical resistivity which is connected with CEF we subtracted the residual resistivity and phononic contribution in a similar manner as in [2]. We used for the subtraction of phononic term the Bloch-Grüneisen formula for electron-phonon scattering $\rho_{\mathrm{ph}}(T)$. We obtained the best results with the Debye temperature $\Theta_{\mathrm{D}}=380 \mathrm{~K}$. The magnetic contribution $\rho_{\mathrm{mag}}(T)$ (Fig. 2) is anisotropic and it follows the characteristic shape [2] with high temperature saturation $\left(\rho_{\text {mag }}^{a}(300 \mathrm{~K})=4.5 \mu \Omega \mathrm{cm}\right.$, $\rho_{\mathrm{mag}}^{b}(300 \mathrm{~K})=4.9 \mu \Omega \mathrm{cm}$ and $\left.\rho_{\mathrm{mag}}^{c}(300 \mathrm{~K})=1.8 \mu \Omega \mathrm{cm}\right)$. All dependences contain the maximum at $T_{\mathrm{N}}$. This behaviour is characteristic of the scattering of the conduction electrons on the disordered magnetic moments (spin disorder resistivity model [2]) in a presence of the CEF. The anisotropy of the magnetic contribution to the electrical resistivity of $\mathrm{DyNi}_{5}$ is similar to $\mathrm{PrNi}_{5}$ [2] where 
the contribution along $\boldsymbol{c}$ axis is about 2.5 times smaller than along $\boldsymbol{b}$ and $\boldsymbol{a}$. Also $\rho_{\text {mag }}^{\boldsymbol{b}}(T) \geq \rho_{\text {mag }}^{\boldsymbol{a}}(T)$. Due to this behaviour we could expect the anisotropic CEF contribution to the electron-quasiparticle interaction function which has been measured by PC spectroscopy [7] along $a$ axis. This contribution will be smaller along $c$ axis than along $b$ or $a$ axis.

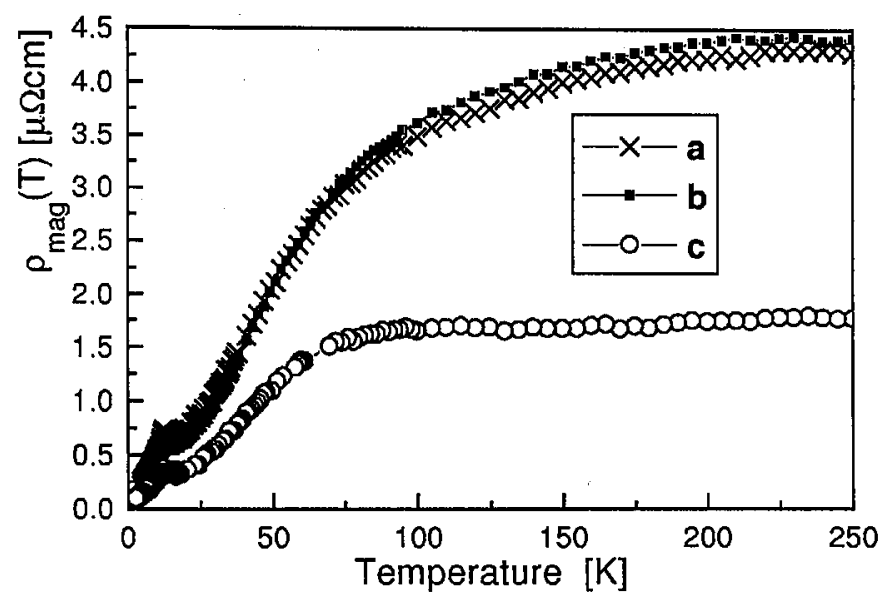

Fig. 2. The magnetic contributions $\rho_{\mathrm{mag}}(T)$ to the the electrical resistivity of single crystal $\mathrm{DyNi}_{5}$ along main crystallographical axes.

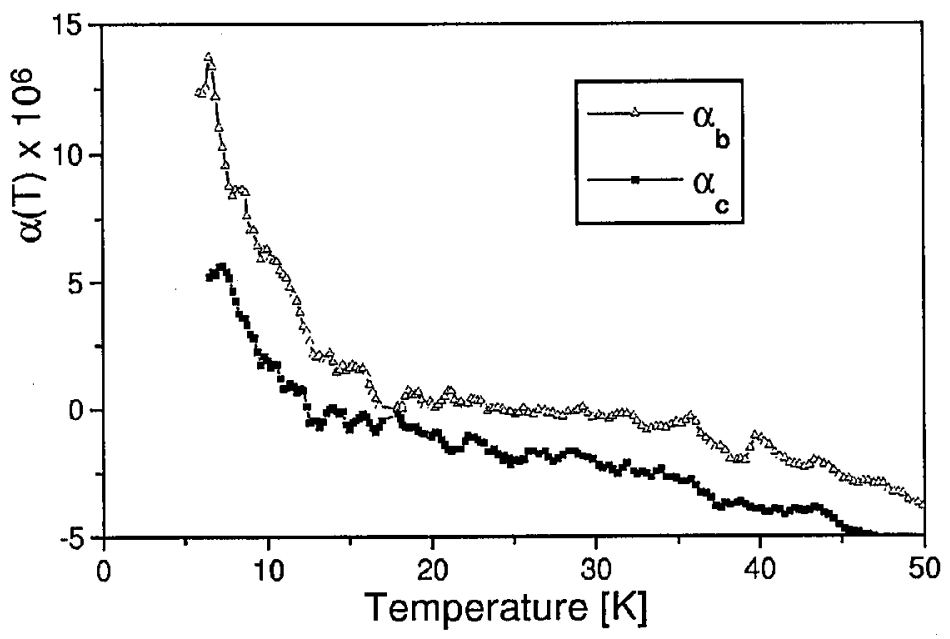

Fig. 3. The temperature dependences of the linear thermal expansions coefficients $\alpha$ perpendicular and parallel to the basal plane of single crystal $\mathrm{DyNi}_{5}$.

The measurements of the temperature dependence of susceptibility along main crystallographical axis were performed in order to check the transition temperature $T_{\mathrm{N}}$ determined from electrical resistivity. We obtained the same value of 
$T_{\mathrm{N}}$ for both methods. The susceptibility data are similar to the previous data [5] and they are characteristic of antiferromagnetic transition. We observed that the $\boldsymbol{c}$ axis is the hard axis of magnetization. The magnitudes of susceptibility in magnetically ordered state along $b$ are slightly higher than along $a$.

In Fig. 3 the low temperature part $(T<30 \mathrm{~K})$ of the linear thermal expansion coefficient $\alpha(T)$ perpendicular $\left(\alpha_{c}(T)\right)$ and parallel $\left(\alpha_{b}(T)\right)$ to basal plane are shown. At low temperatures below $T_{\mathrm{N}}$ both dependences become positive. At high temperatures the $\alpha(T)$ is always negative for both orientation and $\alpha_{b}(T)>\alpha_{c}(T)$. Both dependences saturate to constant value at room temperature $300 \mathrm{~K}\left(\alpha_{b}(T)=\right.$ $-21 \times 10^{-6} \mathrm{~K}^{-1}$ and $\alpha_{c}(T)=-22 \times 10^{-6} \mathrm{~K}^{-1}$ ). On the other hand, one could see the change of slope of both dependences at about $T_{\mathrm{N}}=12.2 \mathrm{~K}$. Generally, the magnitudes of absolute values of $\alpha(T)$ are smaller than in case of $\mathrm{PrNi}_{5}$ [3].

The electrical resistivity, susceptibility and thermal expansion of single crystal $\mathrm{DyNi}_{5}$ along main crystallographical axis were measured. We estimated the anisotropic magnetic contribution to the electrical resistivity and the linear thermal expansion coefficient.

\section{Acknowledgments}

This work was supported, in part, by the Slovak Grant Agency VEGA (grant No. 2/4176/97 and No. 2/5139/98). Liquid nitrogen needed for experiments was sponsored by VS Oce s.r.o., Košice.

\section{References}

[1] J.J.M. Franse, R.J. Radwanski, Handbook of Magnetic Materials, Vol. 7, Ed. K.H.J. Buschow, North-Holland, Amsterdam 1993, p. 307.

[2] J.A. Blanco, M. Reiffers, D. Gignoux, D. Schmitt, A.G.M. Jansen, Phys. Rev. B 44, 9325 (1991).

[3] H.R. Ott, K. Andres, E. Bucher, J.P. Maita, Solid State Commun. 18, 1303 (1976).

[4] R.J. Radwanski, N.H. Kim-Ngan, F.E. Kayzel, J.J.M. Franse, Int. J. Mod. Phys. $B$ 7, 379 (1993).

[5] A. Na it Saida, Ph.D. thesis, Laboratoire Louis Néel Grenoble, Grenoble 1980.

[6] F.E. Kayzel, Ph.D. thesis, University of Amsterdam, Amsterdam 1997.

[7] E. Kacmarciková, T. Salonová, M. Reiffers, M. Mihalik, A.A. Menovsky, J. Magn. Magn. Mater. 196-197, 716 (1999). 